\title{
Fixed Point Theorems for Hybrid Mappings in Metric Spaces
}

\author{
Reny George* ${ }^{*}$, M.S. Khan ${ }^{\uparrow}$, And Abraham Varghese
}

ABSTRACT. Some common fixed point theorems for non-self hybrid mappings have been proved by altering the distance between the points. Our results extends and generalizes many well known results.

\section{INTRODUCTION}

Since the appearance of celebrated Banach contraction principle, sever al generalization of the theorem in the setting of point to point mappings have been obtained. In 1976 Jungck [3] initially gave a common fixed point theorem for commuting mappings which generalized Banach's Fixed Point Theorem. Jungck's theorem was generalized, extended and unified in various ways by many authors. S. Sessa [10] defined a generalization of commuting mappings which is called weakly commuting mappings. Recently Jungck [2] introduced the concept of compatible mappings for single valued mappings which are more general than weakly commuting mappings. Nadler [6] was the first to extend Banach's theorem to multivalued contraction mappings. In the sequel the concept of commuting mappings, weakly commuting mappings and compatible mappings were extended to hybrid mappings (i.e one single valued and one multivalued mappings). Recently, non-linear Hybrid contractions, that is contraction types involving single valued and multivalued mappings have been studied by many authors (see Mukerjee [5], Niampally et al. [7], Sessa et al. [10], Singh et al. [11], Khan et al [4], Rashwan and Ahmed [8]). Rashwan and Ahmed [8], Delbosco [1] and skof [1] proved some fixed point theorems for self maps of complete metric spaces by altering the distance between the points with the use of a function $\phi:[0, \infty) \longrightarrow[0, \infty)$, where $\phi$ satisfy the following properties.

(i) $\phi$ is continuous and strictly increasing in $[0, \infty)$

(ii) $\phi(t)=0$ iff $t=0$

2000 Mathematics Subject Classification. Primary: 54H25.

Key words and phrases. Hybrid Mapping, contractive type mappings, compatible mappings, common fixed points, Multivalued mappings.

${ }^{*}$ Research supported by University Grants Commission C.R.O, Bhopal, India (Grant No. 4126(8)/98(MRP/CRO))

${ }^{\uparrow}$ Corresponding author 
(iii) $\phi(t) \geq M . t^{\mu}$ for every $t>0$, where $M>0, \mu>0$ are constants.

Rashwan and Ahmed [8], proved the following:

Theorem 1.1. Let $f$ be a mapping of a complete metric space $(X, d)$ into itself and let $S: X \longrightarrow B(X)$ be such that

1.1.1) $S(X) \subset f(X)$

1.1.2) $f$ is continuous

1.1.3) $S$ and $f$ are $\delta$-compatible.

1.1.4) $f(K)$ is complete

1.1.4) $\phi(\delta(S x, S y)) \leq a . \quad \phi(d(f x, f y))+b\{\phi(D(f x, S x))+\phi(D(f y, S y))\}+$ $c\left\{\frac{\phi(D(f x, S y))+\phi(D(f y, S x))}{2}\right\}$ for all $x, y \in X, x \neq y$, where $a, b$, $c$ are constants satisfying $a+2 b+2 c<1$ and $\phi:] 0, \infty[\longrightarrow] 0, \infty[$ satisfy the property (i), (ii) and (iii).

Then $S$ and $f$ have a unique common fixed point $u$ in $X$. Moreover $S u=\{u\}=$ $\{f u\}$.

In this paper we prove a common fixed point theorem for $\delta$-compatible mappings, by altering the distance between points, using function $\phi$ which satisfies conditions (i), (ii) and

(iv) $\phi$ is bijective.

Our results generalizes and extends the results of Rashwan and Ahmed [8], Delbosco [1], Skof [12] and many others.

\section{Preliminaries}

Let $(X, d)$ be a complete metric space and let $B(X)$ be the set of all nonempty, bounded subsets of $X$. For any $A, B \in B(X)$, we have $\delta(A, B)=\sup \{d(a, b)$ : $a \in A, b \in B\}$ and $D(A, B)=\inf \{d(a, b): a \in A, b \in B\}$. If $A$ consists of a single point $a$, we write,

$$
\delta(a, B)=\sup \{d(a, b): b \in B\}
$$

Remark 2.1. Note that if $\phi:[0, \infty) \longrightarrow[0, \infty)$ satisfies (i), (ii) and (iv) above and if $d$ is a metric defined on $X$, then the composition $\phi \circ d=\phi(d)$ is not necessarily a metric as shown in the following:

Example 2.1. Let $X=\{a, b, c\}$ and $d: X \times X \longrightarrow R$ be given by $d(a, b)=$ $d(b, a)=1, d(b, c)=d(c, b)=1$ and $d(a, c)=d(c, a)=2$ and $\phi$ be given by $\phi(t)=t^{2}$ for all $t \in[0, \infty)$. Then clearly $d$ is a metric on $X$ but $\phi(d(a, c))>$ $\phi(d(a, b)+d(b, c))$.

We also remark that if $\phi$ is increasing then so is $\phi^{-1}$.

We say that the subset $A$ of $X$ is the limit of a sequence $\left\{A_{n}\right\}, n=1,2,3, \ldots$ of nonempty subsets of $X$ iff

1) each point $a$ in $A$ is the limit of a sequence $\left\{a_{n}\right\}$ with $a_{n}$ in $A_{n}$ for $n=$ $1,2,3 \ldots$ 
2) for arbitrary $\epsilon>0$ there exists an integer $N$ such that $A_{n} \subset A_{\epsilon}$ for $n \geq N$, where $A_{\epsilon}$ is the union of all open spheres with centers in $A$ and radius $\epsilon$.

The following definition is due to Jungck-Rhoades [2]:

Definition 2.1. Let $(X, d)$ be a metric space. Let $f: X \longrightarrow X$ and $S: X \longrightarrow$ $B(X)$. $S$ and $f$ are $\delta$-compatible iff $f S x \subset B(X)$ for $x \in X$ and $\delta(S f x n, f S x n)=$ 0 whenever $\{x n\}$ is a sequence in $X$ such that $f x n \rightarrow t$ and $S x n \rightarrow\{t\}$ for some $t \in X$.

Proposition $2.1([2])$. Let $(X, d)$ be a complete metric space. Suppose $f: X \longrightarrow$ $X, S: X \longrightarrow B(X)$, and $f$ and $S$ are $\delta$-compatible

a) Suppose that the sequence $\{S x n\}$ converges to $\{u\}$ and $\{f x n\}$ converges to $u$. If $f$ is continuous, then $S f x n \rightarrow\{f u\}$.

b) If $\{f u\}=S u$ for some $u \in X$, then $S f u=f S u$.

\section{Main Results}

Theorem 3.1. Let $(X, d)$ be a metric space, $K$ be a non-empty, closed and bounded subset of $X$.

Let $S, T: K \longrightarrow C B(X)$ and $f: K \longrightarrow X$ be such that

3.1.1) $S(K) \cup T(K) \subset f(K)$

3.1.2) $f$ is continuous

3.1.3) the pairs $(S, f)$ and $(T, f)$ are $\delta$-compatible

3.1.4) $f(K)$ is complete

3.1.5) $\phi(\delta(S x, T y)) \leq a \cdot \phi(d(f x, f y))+b \cdot\{\phi(\delta(f x, S x))+\phi(\delta(f y, T y))\}+$ $c\{\phi(\delta(f x, T y))+\phi(\delta(f y, S x))\}$ for all $x, y \in K, x \neq y$, where $a, b, c$ are constants satisfying $a+2 b+2 c<1$ and $\phi:[0, \infty) \longrightarrow[0, \infty)$ satisfy the property (i), (ii) and (iv).

Then $S, T, f$ have a unique common fixed point $u$ in $X$. Moreover $S u=T u=$ $\{u\}=\{f u\}$.

Proof. Let $x_{0} \in K$ be an arbitrary point in $K$ and define the sequence $\left\{x_{n}\right\}$ as follows. Take a point $x_{1}$ in $K$ such that $f x_{1} \in S x_{0}=X_{0}$, and then take $x_{2}$ in $K$ such that $f x_{2} \in T x_{1}=X_{1}$. Proceeding this way, we get sequence $\left\{x_{n}\right\}$ in $K$ and $\left\{f x_{n}\right\}$ in $f(K)$ such that

$$
\begin{aligned}
& f x_{2 n+1} \in S x_{2 n}=X_{2 n} \\
& f x_{2 n+2} \in T x_{2 n+1}=X_{2 n+1}, \quad n=0,1,2, \ldots
\end{aligned}
$$


Let $\delta_{n}=\delta\left(X_{n}, X_{n+1}\right), n=0,1,2, \ldots$ Then we have

$$
\begin{aligned}
\phi\left(\delta_{2 n+2}\right) & =\phi\left(\delta\left(S x_{2 n+2}, T x_{2 n+3}\right)\right) \leq \\
& \leq a \cdot \phi\left(d\left(f x_{2 n+2}, f x_{2 n+3}\right)\right)+ \\
& +b \cdot\left\{\phi\left(\delta\left(f x_{2 n+2}, S x_{2 n+2}\right)\right)+\phi\left(\delta\left(f x_{2 n+3}, T x_{2 n+3}\right)\right)\right\}+ \\
& +c \cdot\left\{\frac{\phi\left(\delta\left(f x_{2 n+2}, T x_{2 n+3}\right)\right)+\phi\left(\delta\left(f x_{2 n+3}, S x_{2 n+2}\right)\right)}{2}\right\} \leq \\
& \leq a \cdot \phi\left(\delta\left(T x_{2 n+1}, S x_{2 n+2}\right)\right)+ \\
& +b \cdot\left\{\phi\left(\delta\left(T x_{2 n+1}, S x_{2 n+2}\right)\right)+\phi\left(\delta\left(S x_{2 n+2}, T x_{2 n+3}\right)\right)\right\}+ \\
& +c \cdot\left\{\frac{\phi\left(\delta\left(T x_{2 n+1}, T x_{2 n+3}\right)\right)+\phi\left(\delta\left(S x_{2 n+2}, S x_{2 n+2}\right)\right)}{2}\right\} \leq \\
& \leq a \cdot \phi\left(\delta_{2 n+1}\right)+b \cdot\left(\phi\left(\delta_{2 n+1}\right)+\phi\left(\delta_{2 n+2}\right)\right)+ \\
& +c \cdot\left\{\frac{\phi\left(\delta_{2 n+1}\right)+\phi\left(\delta_{2 n+2}\right)+\phi\left(\delta_{2 n+1}\right)+\phi\left(\delta_{2 n+1}\right)}{2}\right\}
\end{aligned}
$$

Hence we have

$$
(1-b-c / 2) \cdot \phi\left(\delta_{2 n+2}\right) \leq(a+b+3 c / 2) \cdot \phi\left(\delta_{2 n+1}\right.
$$

i.e.

$$
\phi\left(\delta_{2 n+2}\right) \leq\left(\frac{a+b+3 c / 2}{1-b-c / 2}\right) \cdot \phi\left(\delta_{2 n+1}\right)<\phi\left(\delta_{2 n+1}\right)
$$

Also,

$$
\begin{aligned}
\phi\left(\delta_{2 n+1}\right) & =\phi\left(\delta\left(S x_{2 n+2}, T x_{2 n+1}\right)\right) \leq \\
& \leq a \cdot \phi\left(d\left(f x_{2 n+2}, f x_{2 n+1}\right)+\right. \\
& +b \cdot\left\{\phi\left(\delta\left(f x_{2 n+2}, S x_{2 n+2}\right)\right)+\phi\left(\delta\left(f x_{2 n+1}, T x_{2 n+1}\right)\right)\right\}+ \\
& +c\left\{\frac{\phi\left(\delta\left(f x_{2 n+2}, T x_{2 n+1}\right)\right)+\phi\left(\delta\left(f x_{2 n+1}, S x_{2 n+2}\right)\right)}{2}\right\} \leq \\
& \leq a \cdot \phi\left(\delta\left(T x_{2 n+1}, S x_{2 n}\right)\right)+ \\
& +b \cdot\left\{\phi\left(\delta\left(T x_{2 n+1}, S x_{2 n+2}\right)\right)+\phi\left(\delta\left(S x_{2 n}, T x_{2 n+1}\right)\right)\right\}+ \\
& +c \cdot\left\{\frac{\phi\left(\delta\left(T x_{2 n+1}, T x_{2 n+1}\right)\right)+\phi\left(\delta\left(S x_{2 n}, S x_{2 n+2}\right)\right)}{2}\right\} \leq \\
& \leq a \cdot \phi\left(\delta_{2 n}\right)++b \cdot\left(\phi\left(\delta_{2 n+1}\right)+\phi\left(\delta_{2 n}\right)\right)+ \\
& +c \cdot\left\{\frac{\phi\left(\delta_{2 n+1}\right)+\phi\left(\delta_{2 n}\right)+\phi\left(\delta_{2 n}\right)+\phi\left(\delta_{2 n}\right)}{2}\right\}
\end{aligned}
$$

Hence we have

$$
(1-b-c / 2) \phi\left(\delta_{2 n+1}\right) \leq(a+b+3 c / 2) \phi\left(\delta_{2 n}\right)
$$


i.e.

$$
\phi\left(\delta_{2 n+1}\right) \leq\left(\frac{a+b+3 c / 2}{1-b-c / 2}\right) \phi\left(\delta_{2 n}\right)<\phi\left(\delta_{2 n}\right)
$$

From (1) and (2) we get,

$$
\phi\left(\delta_{n+1}\right) \leq\left(\frac{a+b+3 c / 2}{1-b-c / 2}\right) \phi\left(\delta_{n}\right)<\phi\left(\delta_{n}\right)
$$

Since $\phi$ is increasing, we have $\delta_{n+1}<\delta_{n}$. Therefore $\left\{\delta_{n}\right\}$ is a decreasing sequence which has a limit $\delta$.

Letting $n \rightarrow \infty$ in (3) we get

$$
\phi(\delta) \leq\left(\frac{a+b+3 c / 2}{1-b-c / 2}\right) \phi(\delta)<\phi(\delta),
$$

a contradiction.

Hence $\phi(\delta)=0 \Longrightarrow \delta=0$

Let $z_{n}$ be an arbitrary point in $X_{n}$ for $n=0,1,2, \ldots$ We have $\lim _{n \rightarrow \infty} d\left(z_{n}\right.$, $\left.z_{n+1}\right)=\lim _{n \rightarrow \infty} \delta\left(X_{n}, X_{n+1}\right)=0$.

We will claim that sequence $\left\{z_{n}\right\}$ is a Cauchy sequence. Suppose that $\left\{z_{n}\right\}$ is not a Cauchy sequence, then there exists a positive number $\epsilon$ such that for each positive integer $k$, there exists integers $n(k)$ and $m(k)$ such that

$$
k \leq n(k)<m(k)
$$

and $d\left(z_{n(k)}, z_{m(k)}\right) \geq \epsilon$, i.e.

$$
\delta\left(X_{n(k)}, X_{m(k)}\right) \geq \epsilon
$$

Thus for each integer $k$ we have,

$$
\epsilon \leq \delta\left(X_{n(k)}, X_{m(k)}\right) \leq \delta\left(X_{n(k)}, X_{m(k)-1}\right)+\delta\left(X_{m(k)-1}, X_{m(k)}\right) .
$$

For each integer $k$, let $m(k)$ denote the smallest integer satisfying (4) and (5) for some $n(k)$. Then we have $\delta\left(X_{n(k)}, X_{m(k)-1}\right)<\epsilon$ and it follows from (6) that

$$
\lim _{k \rightarrow \infty} \delta\left(X_{n(k)}, X_{m(k)}\right) \leq \epsilon
$$

Using the triangle inequality, we get

$$
\mid \delta\left(X_{n(k)}, X_{m(k)-1)}-\delta\left(X_{n(k)}, X_{m(k)}\right) \mid \leq \delta\left(X_{m(k)-1}, X_{m(k)}\right)\right.
$$

and

$$
\mid \delta\left(X_{n(k)+1}, X_{m(k)-1)}-\delta\left(X_{n(k)}, X_{m(k)}\right) \mid \leq \delta\left(X_{n(k)}, X_{n(k)+1}\right)+\delta\left(X_{m(k)-1}, X_{m(k)}\right)\right.
$$

which yield

$$
\lim _{k \rightarrow \infty} \delta\left(X_{n(k)}, X_{m(k)-1}\right) \leq \lim _{k \rightarrow \infty} \delta\left(X_{n(k)+1}, X_{m(k)-1}\right) \leq \epsilon .
$$


Now

$$
\begin{aligned}
\phi\left(\delta\left(X_{n(k)}, X_{m(k)}\right)\right) & \leq \phi\left(\delta\left(X_{n(k)}, X_{n(k)+1}\right)+\left(\delta\left(X_{n(k)+1}, X_{m(k)}\right)\right)=\right. \\
& =\phi\left(\delta\left(X_{n(k)}, X_{n(k)+1}\right)+\delta\left(S x_{m(k)}, T x_{n(k)+1}\right)\right) \leq \\
& \leq \phi\left(\left(\delta\left(X_{n(k)}, X_{n(k)+1}\right)+\phi^{-} 1\left(a \cdot \phi\left(d\left(f x_{m(k)}, f x_{n(k)+1}\right)\right)+\right.\right.\right. \\
& +b\left\{\phi\left(\delta\left(f x_{m(k)}, S x_{m(k)}\right)\right)+\phi\left(\delta\left(f x_{n(k)+1}, T x_{n(k)+1}\right)\right)\right\}+ \\
& +c\left\{\frac{\phi\left(\delta\left(f x_{m(k)}, T x_{n(k)+1}\right)\right)+\phi\left(\delta\left(f x_{n(k)+1}, S x_{m(k)}\right)\right)}{2}\right\} \leq \\
& \leq \phi\left(\left(\delta\left(X_{n(k)}, X_{n(k)+1}\right)+\phi^{-1}\left(a \cdot \phi\left(\delta\left(X_{m(k)-1}, X_{n(k)}\right)\right)+\right.\right.\right. \\
& +b\left\{\phi\left(\delta\left(X_{m(k)-1}, X_{m(k)}\right)\right)+\phi\left(\delta\left(X_{n(k)}, X_{n(k)+1}\right)\right)\right\}+ \\
& +c\left\{\frac{\phi\left(\delta\left(X_{m(k)-1}, X_{n(k)+1}\right)\right)+\phi\left(\delta\left(X_{n(k)}, X_{m(k)}\right)\right)}{2}\right\}
\end{aligned}
$$

Letting $k \rightarrow \infty$ we get,

$$
\phi(\epsilon)=\phi\left(0+\phi^{-1}\left(a \cdot \phi(\epsilon)+0+c \cdot\left(\frac{\phi(\epsilon)+\phi(\epsilon)}{2}\right)\right)\right)
$$

i.e. $\phi(\epsilon) \leq(a+c) \cdot \phi(\epsilon)<\phi(\epsilon)$, a contradiction. Hence $\left\{z_{n}\right\}$ is a Cauchy sequence. Therefore the sequence $\left\{f x_{n}\right\}$ is also a cauchy sequence in $f(K)$ and hence converges to some point say $z$ in $f(K)$. Let $u \in \phi^{-1} z$. Thus there exists $u$ in $K$ such that $f u=z$. The subsequences $\left\{f x_{2 n}\right\},\left\{f x_{2 n+1}\right\}$ will also converge to $z$ and the sequence of the sets $\left\{S x_{2 n}\right\},\left\{T x_{2 n+1}\right\}$ will converge to $\{z\}$. Since $f$ is continuous, $f^{2} x_{2 n} \rightarrow f z, f^{2} x_{2 n+1} \rightarrow f z, f S x_{2 n} \rightarrow\{f z\}, f T x_{2 n+1} \rightarrow\{f z\}$. But the pairs $(S, f)$ and $(T, f)$ are $d$-compatible, therefore $S f x_{2 n} \rightarrow\{f z\}, T f x_{2 n+1} \rightarrow\{f z\}$.

Now

$$
\begin{aligned}
\phi\left(\delta\left(S f x_{2 n}, T x_{2 n+1}\right)\right) & \leq a \cdot \phi\left(d\left(f^{2} x_{2 n}, f x_{2 n+1}\right)\right)+ \\
& +b \cdot\left\{\phi\left(\delta\left(f^{2} x_{2 n}, S f x_{2 n}\right)\right)+\phi\left(\delta\left(f x_{2 n+1}, T x_{2 n+1}\right)\right)\right\}+ \\
& +c \cdot\left\{\frac{\phi\left(\delta\left(f^{2} x_{2 n}, T x_{2 n+1}\right)\right)+\phi\left(\delta\left(f x_{2 n+1}, S f x_{2 n}\right)\right)}{2}\right\}
\end{aligned}
$$

Letting $n \rightarrow \infty$, we get

$$
\begin{aligned}
\phi(d(f z, z))= & a \cdot \phi(d(f z, z))+b \cdot\{\phi(d(f z, f z))+\phi(d(z, z))\}+ \\
& +c \cdot\left\{\frac{\phi(d(f z, z))+\phi(d(z, f z))}{2}\right\}= \\
= & (a+c) \cdot \phi(d(f z, z))<\phi(d(f z, z))
\end{aligned}
$$

a contradiction. Hence $f z=z$. 
Again we have

$$
\begin{aligned}
\phi\left(\delta\left(S z, T x_{2 n+1}\right)\right) & =a \cdot \phi\left(d\left(f z, f x_{2 n+1}\right)\right)+ \\
& +b \cdot\left\{\phi(\delta(f z, S z))+\phi\left(\delta\left(f x_{2 n+1}, T x_{2 n+1}\right)\right)\right\}+ \\
& +c \cdot\left\{\frac{\phi\left(\delta\left(f z, T x_{2 n+1}\right)\right)+\phi\left(\delta\left(T x_{2 n+1}, S z\right)\right)}{2}\right\}
\end{aligned}
$$

Letting $n \rightarrow \infty$, we get

$$
\begin{aligned}
\phi(\delta(S z, z)) & \leq a \cdot \phi(0)+b \cdot\{\phi(\delta(z, S z))+\phi(0)\}+c \cdot\left\{\frac{\phi(0)+\phi(\delta(z, S z))}{2}\right\}= \\
& =(b+c / 2) \cdot \phi(\delta(S z, z))<\phi(\delta(S z, z)),
\end{aligned}
$$

a contradiction, hence $S z=\{z\}$.

Similarly

$$
\begin{aligned}
\phi\left(\delta\left(T z, S x_{2 n}\right)\right) & \leq a \cdot \phi\left(d\left(f x_{2 n}, f z\right)\right)+b \cdot\left\{\phi\left(\delta\left(f x_{2 n}, S x_{2 n}\right)\right)+\phi(\delta(f z, T z))\right\}+ \\
& +c \cdot\left\{\frac{\phi\left(\delta\left(f z, S x_{2 n}\right)\right)+\phi\left(\delta\left(T z, f x_{2 n}\right)\right)}{2}\right\}
\end{aligned}
$$

Letting $n \rightarrow \infty$, we get

$$
\begin{aligned}
\phi(\delta(T z, z)) & \leq a \cdot \phi(0)+b \cdot\{\phi(0)+\phi(\delta(z, T z))\}+c \cdot\left\{\frac{\phi(0)+\phi(\delta(z, T z))}{2}\right\}= \\
& =(b+c / 2) \phi(\delta(T z, z))<\phi(\delta(T z, z)),
\end{aligned}
$$

a contradiction. Hence $T z=\{z\}$.

Now let $v \in X$ be a common fixed point of $S, T$ and $f, v \neq z$. Then

$$
\begin{aligned}
\phi(d(v, z)) & \leq \phi(\delta(S z, T v)) \\
& \leq a \cdot \phi(d(f z, f v))+b \cdot\{\phi(\delta(f z, S z))+\phi(\delta(f v, T v))\}+ \\
& +c \cdot\left\{\frac{\phi(\delta(f v, S z))+\phi(\delta(f z, T v))}{2}\right\} \leq \\
& \leq a \cdot \phi(d(z, v))+b \cdot(\phi(0)+\phi(0))+c \cdot\left\{\frac{\phi(d(v, z))+\phi(d(z, v))}{2}\right\}= \\
& =(a+c) \cdot \phi(d(z, v))<\phi(d(z, v)),
\end{aligned}
$$

a contradiction. Hence $v=z$ and $z$ is the unique common fixed point of $S, T$ and $f$.

In Theorem 3.1 if we put $S=T$ we get following:

Corollary 3.1. Let $f$ be a mapping of a complete metric space $(X, d)$ into itself and let $S: X \longrightarrow B(X)$ be such that

3.2.1) $S(X) \subset f(X)$

3.2.2) $f$ is continuous

3.2.3) $S$ and $f$ are $\delta$-compatible.

3.2.4) $f(K)$ is complete 
3.2.5) $\phi(\delta(S x, S y)) \leq a \cdot \phi(d(f x, f y))+b\{\phi(\delta(f x, S x))+\phi(\delta(f y, S y))\}+$ $c\left\{\frac{\phi(\delta(f x, S y))+\phi(\delta(f y, S x))}{2}\right\}$ for all $x, y \in X, x \neq y$, where $a, b, c$ are constants satisfying $a+2 b+2 c<1$ and $\phi:] 0, \infty[\longrightarrow] 0, \infty[$ satisfy the property (i), (ii) and (iv).

Then $S$ and $f$ have a unique common fixed point $u$ in $X$. Moreover $S u=\{u\}=$ $\{f u\}$.

Remark 3.1. Since $d(a, B) \leq \delta(a, B)$, we see that the above corollary is a substantial generalization and extension of [Theorem 3.1,8] .

Assuming $f$ to be the identity mapping in Corollary 1 we get the following:

Corollary 3.2. Let $S$ be a mapping of a complete metric space $(X, d)$ into itself such that

$$
\begin{aligned}
\text { 3.3.1) } & S(X) \subseteq X \\
3.3 .2) & \phi(\delta(S x, S y)) \leq a \cdot \phi(d(f x, f y))+b\{\phi(\delta(f x, S x))+\phi(\delta(f y, S y))\}+ \\
& +c\left\{\frac{\phi(\delta(f x, S y))+\phi(\delta(f y, S x))}{2}\right\} \text { for all } x, y \in X, x \neq y, \text { where } a, b, \\
& c \text { are constants satisfying } a+2 b+2 c<1 \text { and } \phi:] 0, \infty[\longrightarrow] 0, \infty[\text { satisfy } \\
& \text { the property (i), (ii) and (iv). }
\end{aligned}
$$

Then $S, f$ have a unique common fixed point $u$ in $X$.

Remark 3.2. Corollary 3.3 is a proper generalization of the corresponding result of $[12]$.

\section{An application.}

We now apply Theorem 3.1 to prove a common fixed point theorem satisfying a contraction condition more general than (3.1.5) and under some compactness type condition.

Theorem 3.2. Let $(X, d)$ be a metric space, $K$ be a compact subset of $X$. Let $S, T: K \longrightarrow C B(X)$ and $f: K \longrightarrow X$ be such that

$$
\text { 3.4.1) } S(K) \bigcup T(K) \subset f(K)
$$

3.4.2) $f$ is continuous

3.4.3) the pairs $(S, f)$ and $(T, f)$ are d-compatible

3.4.5) $f(K)$ is complete

3.4.5) $\phi(\delta(S x, T y))<a \cdot \phi(d(f x, f y))+b\{(\phi(\delta(f x, S x))+\phi(\delta(f y, T y))\}+$ $+c\left\{\frac{\phi(\delta(f x, T y))+\phi(\delta(f y, S x))}{2}\right\}$ for all $x, y \in K, x \notin y$, with right hand side not zero, where $a, b, c$ are constants satisfying $a+2 b+2 c \leq 1$ and $\phi:(0, \infty[\longrightarrow(0, \infty[$ satisfy the property (i), (ii) and (iv).

Then $S, T$ and $f$ have a unique common fixed point $u$ in $X$. Moreover $S u=$ $T u=\{u\}=\{f u\}$.

Proof. If $a+2 b+2 c<1$, then the proof follows from Theorem 3.1. Suppose $a+2 b+2 c=1$. 
Let

$$
\begin{aligned}
M(x, y)= & a \cdot \phi(d(f x, f y))+b\{(\phi(\delta(f x, S x))+\phi(\delta(f y, T y))\}+ \\
& +c\left\{\frac{\phi(\delta(f x, T y))+\phi(\delta(f y, S x))}{2}\right\}
\end{aligned}
$$

Consider the function $T: K \times K \longrightarrow[0,1)$ defined by

$$
T(x, y)=\frac{\phi(\delta(S x, T y))}{M(x, y)}
$$

for all $x, y \in K$.

Clearly the function $T$ is well defined. Since $K$ is compact, $T$ attains its maximum on $K \times K$ at some point say $(u, v) \in K^{2}$. Let $T(u, v)=c$. Then we have

$$
\frac{\phi(\delta(S x, T y))}{M(x, y)} \leq T(u, v)=c
$$

i.e.

$$
\begin{aligned}
\phi(\delta(S x, T y)) \leq & c \cdot M(x, y)= \\
= & a^{\prime} \cdot \phi(d(f x, f y))+b^{\prime}\{(\phi(\delta(f x, S x))+\phi(\delta(f y, T y))\}+ \\
& +c^{\prime} \cdot\left\{\frac{\phi(\delta(f x, T y))+\phi(\delta(f y, S x))}{2}\right\}
\end{aligned}
$$

for all $x, y \in K, a^{\prime} \geq 0, b^{\prime} \geq 0, c, \geq 0$ and $a^{\prime}+2 b^{\prime}+2 c^{\prime}=c \cdot(a+2 b+2 c)=c<1$. Also since $K$ is compact it is closed and bounded, and thus all conditions of Theorem 3.1 is satisfied and hence by Theorem 3.1, S, T and $f$ has a unique common fixed point.

\section{REFERENCES}

[1] Delbosco, Un'estenione di un teorema sul punto fisso di S. Reih, Sem. Mat. UniversPoliteon. Torino, 35 (1976-77), 233-238.

[2] G. Jungck and B.E. Rhoades, Some fixed point theorems for compatible maps, Intenat. J.math. 7 Math. Sci., 16(3) (1993), 417-428.

[3] G. Junck, Commuting maps and fixed points, Amer. Math. Monthly, 83 (1976), 261-263.

[4] Khan M. S, Y. J. Cho, W. T. Park and M. Mumtaz, Coincidence and common fixed points of hybrid contractions, J. Austral, Math. Son. (Series A) 55 (1993), 369-385.

[5] R.N. Mukherjee., On fixed points of single and set-valued mappings, J. Indian Acad. Math., 4 (1982), 101-103.

[6] N.B. Nadler Jr., Multivalued contraction mappings, Pacific J. Math., 30 (1969), 475-488.

[7] Naimpally S.N, S.L. Singh and J.H.M. Whitefield, Coincidence theorems for hybrid contractions, Math. Nachr., 127 (1986), 177-180.

[8] R.A. Rashwan and M.A. Ahmed, Fixed points of single and set-valued mappings, Kyungpook Math. J., 38 (1998), 29-37. 
[9] Rhoades B. E, S. L. Singh and C. Kulshreshtha, Coincidence theorem for some multivalued mappings, Internat. J. Math. 7 Math. Sci., 7 (1984), 429-434.

[10] Sessa. S, R. N. Mukherjee and T. Som, A common fixed point theorem for weakly commuting mappings, Math. Japon., 31 (1986), 235-245.

[11] Singh. S, Ki Sik Ha and Yeol Je Cho, Coincidence and fised points of nonlinear hybtid contractions, Internat. J. Math. and Math. Sci., 2(2) (1989), 247-256.

[12] F. Skof., Theoremi di punto fisso per applicazioni negli spazi metrici, Atti. Accad. Sci. Torino Cl. Sci. Fis. Mat. Natur, 111(3-4) (1977), 323-329.

Current address:

Department of Mathematics

Eritrea Institute of Technology

C/O Ministry of Education

P.O Box 1056, Asmara

Eritrea

P.G. Department of Mathematics

And Computer Science

St. Thomas College

Ruabandha,Bhilai

Durg (Dist.), Chhattisgarh State

INDIA

E-mail address: renygeorge02@yahoo.com

Department of Mathematics AND STATistics

College of Science,

Sultan Quaboos University

P.O Box 36, Al-Khoud 123

Muscat, Sultanate of Oman

E-mail address: mohammad@squ.edu.om

Department of Mathematics

M.P.C.C.E.T

Kailash Nagar, Bhilai Durg (Dist.), ChHattisgarh State

INDIA

E-mail address: abrahamvarghese77@yahoo.com 